\title{
APPLICATION OF THEORY IN PRACTICE: INTEGRATING AREAS - exact and biological- IN GRADUATE - Study of ECC in hypertensive rats.
}

\author{
Bruna Sabrina Hergert (IC), Ana Gabriela Conceição-Vertamatti (PQ), Larissa Yuri Ishizu (PQ), Luiz \\ Alberto Ramos (PQ), Gustavo Trevisan Costa (IC), Fernando Canova (PQ). Laura Leticia Ramos Rifo \\ $(P Q)$, Dora M. Grassi-Kassisse (PQ). \\ Abstract
}

Exact and Biological sciences are areas of expertise that complement each other. The associations between these areas are little explored in the formation of graduate student. Proposals to recruit students of exact for help with the analysis of the data obtained in Labeest generated great interest of applicants' scholarship BAS-SAE. Data analysis electrocardiography of models of hypertension in rats was carried out by a student of statistics, which can apply their knowledge acquired during their training.

Key words: statistical analyses, biological, rats, hypertension, .

\section{Introduction}

The contribution between the organic areas and accurate is of fundamental importance for the validation of experiments and the dissemination of the same. For statistical validation of some studies there is a need for a large number of experiments. On the other hand, in the Biological area, there are increasing restrictions on the number of animals used, requiring awareness of researchers and awareness in the development of experimental protocols. In this way, this project aimed to find competence in the area of accurate to validate mathematically the results obtained in experimental design with adequate number of animals, $n=6$ in two models of hypertension, induced $(\mathrm{HI})$ and genetics (SHR), per group.

\section{Results and Discussion}

As the experiment has few individuals, the use of frequenter's statistical methodology is inappropriate to present as large sample assumption. Therefore, this work was carried out Bayesian analysis. As there is no information of data distribution, we used the nonparametric technique put the observations. Thus, assuming as the prior distribution uniform that is noninformative, hypothesis tests for mean were applied to two populations for all variables in question. It was considered one-tailed test, namely the alternative hypothesis is larger or smaller, since the goal is to verify that relationship between populations. Consequently, the following results with a high probability (greater than or equal to $85 \%$ ) to the relations given this data set were obtained:

Table: Statistical results of ECG data from hypertensive rats

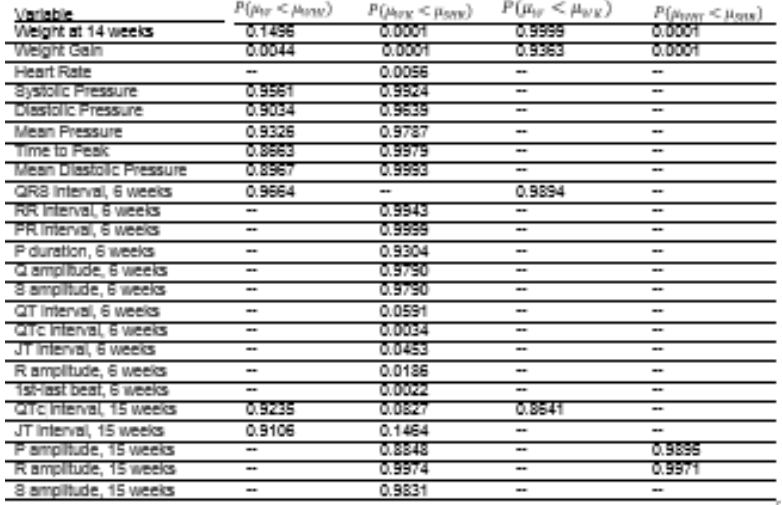

The variables whose values are not included in the table above were not significant in the test. In the case greater than or equal to 0.85 you have written the probability of the alternative hypothesis above is high, therefore, we have smaller values imply higher values for another configuration of the alternative hypothesis, in other words, in the above case we consider the low inequality both types of rats, another option would be the highest ratio. The $\mathrm{HI}$ gained more weight than $\mathrm{W}$, and the WK vs SHR and W as well. The Heart Rate of SHR was higher than the WK. The systolic, diastolic, mean diastolic and mean pressure were higher in $\mathrm{HI}$ and SHR rats that W and WK. Time to peak was higher in $\mathrm{HI}$ and SHR W and WK, indicating left ventricular dyssynchrony. The other indicators show heart and electrolyte changes in SHR and not in HI.

\section{Conclusions}

This draft analysis concluded that the models of hypertension have similar pressure values, though the cardiac function is impaired in the SHR.

\section{Acknowledgement}

Fapesp, Faepex, SAE, CNPq, Capes 